\title{
Diversidad, Composición Y Estructura De Los Sistemas De Producción Agrícolas De Las Comunidades Shuar De La Amazonía Del Ecuador
}

\author{
Hilter Figueroa Saavedra (Ing. Agrónomo .MSc.) \\ Isabel Domínguez Gaibor (Ing. En Ecoturismo .Mgtr.) \\ Universidad / Escuela Superior Politécnica de Chimborazo Extensión Norte \\ Amazónica de la República del Ecuador
}

Doi: 10.19044/esj.2018.v14n21p31 URL:http://dx.doi.org/10.19044/esj.2018.v14n21p31

\begin{abstract}
A diagnosis was applied on agricultural crops in the Shuar Yandia Yacu and San José de Armenia communities, in Orellana (Ecuador). Fourteen farms were chosen, seven in San José de Armenia and seven in Yandia Yacu. For sampling, they were divided into plots of 1x1 m for herbaceous and minor cultivable species, $5 \times 5 \mathrm{~m}$ for shrubs and arable species of medium size, and 10x10 m for bigger cultivable and usable tree species. Data about composition and plant abundance were taken, by direct counting and participatory techniques, such as surveys and interviews. It was identified and classified by crop group, annual, perennial, use and utilitarian value. Indices of richness, diversity, similarity and cumulative frequency of the crops were calculated.

As a result were found 97 agricultural species, the most representative families were Arecaceae, Solanaceae and Fabaceae, mostly fruit trees. The diversity and specific richness was registered low, representing approximately $5 \%$ of the registered agricultural species in the country. The annual food species with the highest accumulated frequency was corn (Zea maize L.), the perennial species of mayor importance was coffee (Coffea arabica L.), and the most important annual ancestral species was yucca (Manihotesculenta Crantz.). In conclusion there is a significant increase of monocultures, therefore it is necessary to recover and strengthen the production of ancestral crops to conserve the diversity of agricultural species in the Amazon region.
\end{abstract}

Keywords: Agro-productive, amazon-region, biodiversity, communities, sustainability. 


\section{Resumen}

Se realizó un diagnóstico de los cultivos agrícolas en las comunidades Shuar: Yandia Yacu y San José de Armenia en la provincia Orellana (Ecuador). Se eligieron 14 fincas, siete en San José de Armenia y siete en Yandia Yacu. Para la toma de muestras se dividieron en parcelas de 1x1 m para herbáceas y especies cultivables menores, $5 \times 5 \mathrm{~m}$ para arbustos y especies cultivables de tamaño medio, y de 10x10 m para especies arbóreas cultivables y aprovechables de mayor tamaño. Se tomaron datos de composición y abundancia vegetal, mediante la técnica de conteo directo y entrevistas semiestructuradas a los productores. Se identificó y clasificó por grupo de cultivo, anual, perenne, uso y valor utilitario. Se calcularon índices de riqueza, diversidad, similitud y frecuencia acumulada de los cultivos. Como resultado se encontraron 97 especies agrícolas, las familias más representativas fueron Arecaceae, Solanaceae y Fabaceae, en su mayoría frutales. La diversidad y riqueza específica fue baja, representando aproximadamente el 5\% de las especies agrícolas registradas en el país. La especie vegetal alimenticia anual con mayor frecuencia acumulada fue el maíz (Zea maíz L.), la especie perenne de mayor importancia fue el café (Coffea arábica L.), y la especie ancestral anual de mayor relevancia fue la yuca (Manihot esculenta Crantz.). En conclusión, existe un alto incremento de monocultivos, por lo que es necesario recuperar y fortalecer la producción de cultivos ancestrales para conservar la diversidad de especies agrícolas en la región amazónica.

Palabras Claves: Agroproductividad, amazonía, biodiversidad,
comunidades, sostenibilidad.

\section{Introduction}

La amazonía suramericana alberga recursos importantes y estratégicos, que incluyen todos los componentes de la diversidad biológica, los mismos que cumplen un rol fundamental en la nueva economía (Nodari y Tomás, 2016). Además de ser fuente de recursos como el agua, la biodiversidad, suelo y subsuelo, que son la base para la sostenibilidad ecológica, permite reducir el uso de insumos externos (Stupino et al., 2014). Es un territorio altamente biodiverso, de los 103 ecosistemas que hay en el mundo, 84 están presentes en la zona andino-amazónica (Camacho, 2009). Es importante señalar que la biodiversidad no está únicamente expresada en la variedad a nivel biológico, sino manifestada en principios de autonomía, conocimiento, identidad, economía y manejo sustentable de los ecosistemas (Gutiérrez et al., 2015).

Actualmente la amazonía afronta graves amenazas frente al desconocimiento de la biodiversidad agrícola, en donde el modelo dominante derivado de la Revolución Verde se ha convertido en una de las principales 
amenazas contra la biodiversidad (Stupino et al., 2014). En las comunidades amazónicas, la incidencia de la colonización y expansión agrícola ha provocado que las técnicas ancestrales de producción vayan en declive, alterando el uso de territorio (Arias-Gutiérrez et al., 2016). Este fenómeno se ha incrementado en los últimos 60 años, en los cuales se evidencia una alteración de los ecosistemas con la implementación de agroecosistemas altamente simplificados, con un reducido número de especies y variedades mejoradas (Stupino et al., 2014), produciendo una pérdida de especies silvestres beneficiosas para su rol ecológico (Nicholls et al., 2017).

La utilización de agroquímicos con alto grado de toxicidad y productos elaborados para el control de plagas, malezas y nutrición de suelos (Nodari y Tomás 2016) ha incidido directamente en la desarticulación de la organización social, producción y tecnología empleada (Camacho, 2009), provocando cambios estructurales y funcionales totalmente diferentes a los ecosistemas naturales y parcialmente modificados (Nicholls et al., 2017). Evidencias históricas señalan que la sofisticación del poblador ancestral se manifestaba en una adecuada organización social y creación de tecnologías que le permitió un manejo adecuado de los recursos naturales y la producción eficiente. Es así que el $40 \%$ de las plantas que consume la humanidad las produjo la ingeniería genética y la biotecnología prehispánica (Camacho, 2009).

El Ecuador posee aproximadamente el $2 \%$ de selva amazónica. Las provincias amazónicas con mayor población rural figuran entre las más pobres del país, en donde el $28 \%$ de habitantes amazónicos corresponde a pueblos y nacionalidades indígenas, que además de poseer una variedad cultural y lingüística, estos pueblos han aprovechado de forma sostenible los recursos de la región, debido a su profundo conocimiento de la biodiversidad y funcionamiento ecosistémico (INEC, 2010). No obstante, las comunidades indígenas viven en medio de la selva con conexiones a poblaciones colonas, lo que ha implicado cambios en las dinámicas económicas, productivas y sociales (Gutiérrez et al., 2015). Es así que estudios anteriores evidencian el establecimiento de sistemas de producción en poblaciones Kichwas asentadas al interior del Parque Nacional Yasuní, con muy poca diversificación y aprovechamiento de los recursos con altas deficiencias en el manejo, bajo rendimiento y productos de baja calidad (Rodríguez y Castillo, 2005), evidenciando la pérdida de biodiversidad y de conocimientos ancestrales en la producción de cultivos, que están siendo reemplazados por otros de valor económico y social.

En este sentido, la conservación y uso sostenible de ecosistemas frágiles como los amazónicos implican un nuevo enfoque en el manejo de agroecosistemas, a través de la recuperación de sistemas y técnicas ancestrales productivas que sean menos invasivas y permitan la conservación de la diversidad de especies y variedades con gran diversidad genética (Somoza et 
al., 2015). Por tanto, el objeto de estudio fue determinar la estructura y composición de la agrobiodiversidad en los sistemas de producción agrícola de las comunidades Shuar en la amazonía ecuatoriana, para desarrollar estrategias que permitan la sostenibilidad y soberanía alimentaria.

\section{Materiales y Métodos}

Para evaluar la biodiversidad vegetal, se aplicó MEDEBIVE, que es una metodología que fundamenta en el análisis integral de los agroecosistemas (Franco, 2003).

\section{Área de Estudio}

El estudio se realizó en la zona alta de la subcuenta del Río Napo, en las comunidades Shuar: San José de Armenia y Yandia, en una superficie aproximada de 800 ha, pertenecientes al cantón Francisco de Orellana, provincia de Orellana (Figura 1).

La altura promedio va de 254 a 300 m.s.n.m. Las coordenadas de la zona de estudio varían entre $0^{\circ} 03^{\prime} 30^{\prime}$ 'S $76^{\circ} 18^{\prime} 00^{\prime} \mathrm{O}$ y $1^{\circ} 04^{\prime} 40^{\prime}$ 'S $76^{\circ} 00^{\prime} 40^{\prime}$ 'O. Posee una temperatura promedio de $26^{\circ} \mathrm{C}$, precipitación entre los 2800 a 4500 $\mathrm{mm}$ al año, siendo mayo y junio los meses con mayor pluviosidad, mientras que agosto, septiembre y octubre presentan menos pluviosidad (GADPO, 2015).
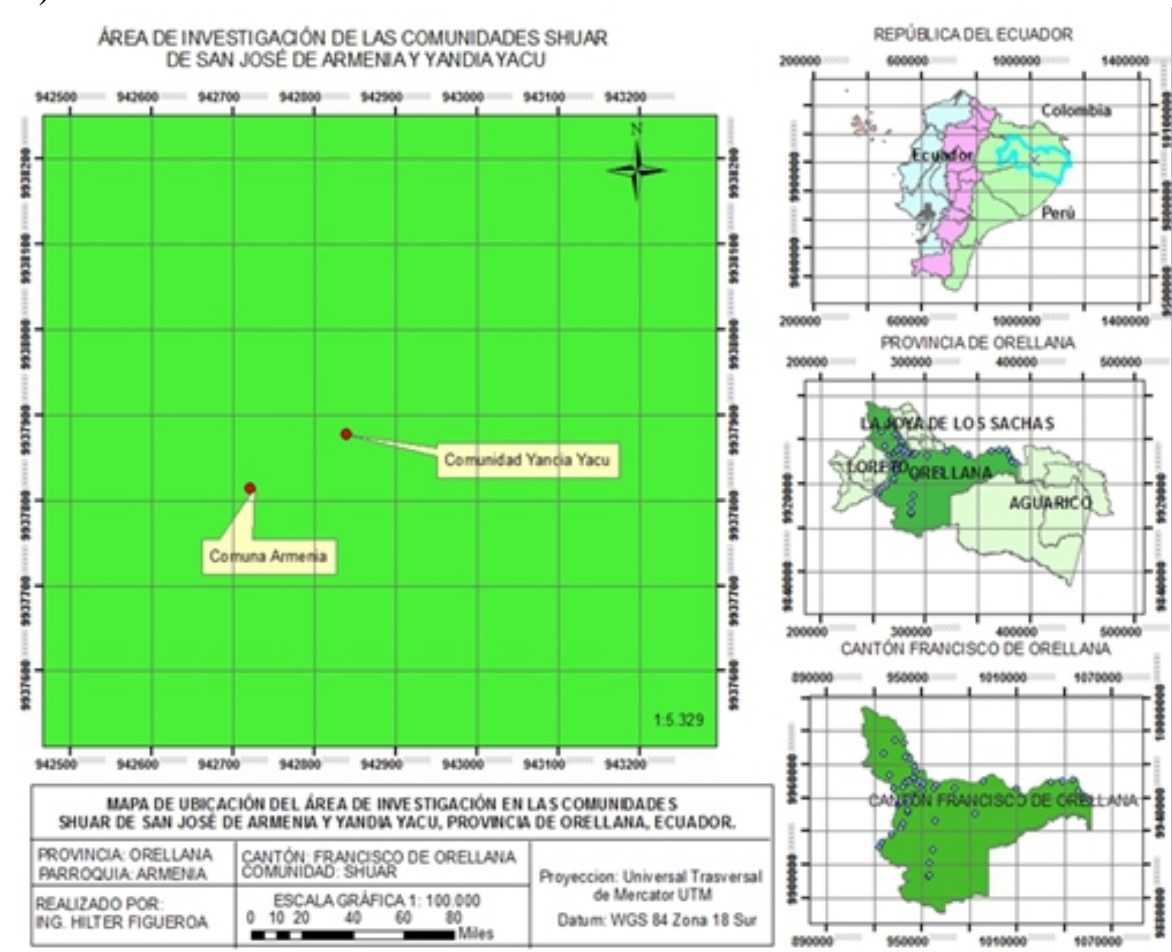

Figura. 1. Área de investigación - Comunidades San José de Armenia y Yandia Yacu. 


\section{Muestreo de Índices Biológicos (Especies Vegetales)}

Se seleccionaron 14 fincas, que corresponden al 10\% de la muestra representativa del área de estudio. Siete fincas pertenecen a la comunidad de San José de Armenia y las otras siete fincas a Yandia Yacu. El estudio se realizó durante el período agosto 2013 y septiembre 2014. En las dos comunidades se identificaron suelos arcillosos y de textura media de color negro, poco profundos con condiciones edáficas similares (GADPO, 2015), por lo que no se evaluó diferencias entre ellos. Para la toma de muestras se consideraron las áreas de cultivos y flora silvestre anual ubicadas en las comunidades señaladas, las que se dividieron en parcelas de $1 \times 1 \mathrm{~m}$ para herbáceas y especies cultivables menores, parcelas de $5 \times 5 \mathrm{~m}$ para arbustos y especies cultivables de tamaño medio, y parcelas de 10x10m para especies arbóreas o especies cultivables y aprovechables de mayor tamaño. Los datos que se tomaron fueron de composición y abundancia vegetal, mediante la técnica de conteo directo, a su vez se aplicaron técnicas participativas como entrevistas semiestructuradas con los productores. Una vez identificadas y clasificadas taxonómicamente las especies, se realizaron inventarios de cultivos agrícolas de acuerdo al grupo de cultivo, anual, perenne, uso y valor utilitario.

\section{Evaluación de la agrobiodiversidad vegetal de los sistemas de producción agrícola de las comunidades San José de Armenia y Yandia Yacu}

Para entender la diversidad y composición vegetal en los diferentes cultivos se calculó la riqueza ( $\mathrm{S}$, número de especies), abundancia y diversidad local por medio del índice de Shannon-Wienner (H') (Hejda et al., 2009) y riqueza de Margalef ( $\mathrm{RMg}$ ). El cálculo del índice de similitud entre las dos comunidades se realizó mediante la ecuación: $\mathrm{S}=2 \mathrm{C} / \mathrm{A}+\mathrm{B}$, donde $\mathrm{A}$ y $\mathrm{B}$ son el número de especies de cada muestra y $\mathrm{C}$ el número de especies comunes en ambas muestras (Lores, 2008). Estos análisis se realizaron en el programa Microsoft Office Excel para Windows, versión 2007 y en el programa DIVERS (Lores, 2008). Para determinar la importancia de las especies se consideró la frecuencia de presencia de cultivos anuales, perennes y forestales en las comunidades estudiadas.

\section{Resultados y Discusión}

\section{Riqueza total de especies agrícolas}

Se encontraron un total de 97 especies agrícolas, 49 familias, 60 de ellas son manejadas por campesinos y de estas, 48 especies son de importancia agrícola, mismas que se encuentran distribuidas en 22 familias. Otras 37 especies son asociadas en su mayoría (arvenses). Las familias con mayor número de especies fueron Arecaceae, Solanaceae y Fabaceae con un 6,12\%, seguidas por Rutacea $5,10 \%$ y Poaceae $3,06 \%$. 


\section{Riqueza de especies por grupo de cultivo}

Uno de los indicadores medidos fue el uso y aprovechamiento de los recursos de agrobiodiversidad. Bajo este parámetro se observa que el grupo de frutales tiene el $49,48 \%$ del total de especies, seguidos por el grupo de uso medicinal con un $22,68 \%$ y para uso como condimento un $14,43 \%$ (Tabla 1 ). Esto se debe a que los frutales están mayormente adaptados a ecosistemas tropicales, además de ser especies perennes que ocupan el suelo por tiempo indefinido y por consiguiente le da mayor estabilidad al sistema agrícola (Lores, 2008).

Tabla 1. Número de especies por grupo de cultivo

\begin{tabular}{|c|c|c|}
\hline Grupo & Especies & \% \\
\hline Frutales & 48 & 49,48 \\
\hline Medicinales & 22 & 22,68 \\
\hline Condimentos & 14 & 14,43 \\
\hline Leguminosas & 6 & 6,19 \\
\hline Tubérculos & 5 & 5,15 \\
\hline Gramíneas & 2 & 2,06 \\
\hline Total de especies & 97 & 100 \\
\hline
\end{tabular}

\section{Índice de Similitud}

Se observó al comparar las fincas de las dos comunidades un índice de similitud promedio de 0,59 , lo cual corresponde a un resultado medio - alto, debido a que en agroecosistemas campesinos el promedio del índice es de 0,70 (Lores, 2008) (Tabla 2).

Tabla 2. Agrobiodiversidad compartida en las comunidades Shuar

\begin{tabular}{|l|l|l|l|l|}
\hline \multicolumn{1}{|c|}{ Familia } & Especie & Nombre Común & \multicolumn{1}{c|}{ Tipo } & Uso \\
\hline Poaceae & Zea mays & Maíz & Gramínea & Alimentación \\
\hline Euphorbiaceae & Manihot sp & Yuca & Tubérculo & Alimentación \\
\hline Musaceae & Mussa sp & Plátano & Frutales & Alimentación \\
\hline Poaceae & Saccharum officinarum & Caña de azúcar & Planta con tallo & Alimentación \\
\hline Fabaceae & Phaseolus sp & Fréjol & Leguminosa & Alimentación \\
\hline Rubiaceae & Coffea sp & Café & Frutales & Alimentación \\
\hline Rubiaceae & Theobroma cacao & Cacao & Frutales & Alimentación \\
\hline Caricaceae & Carica papaya & Papaya & Frutales & Alimentación \\
\hline Arecaceae & Elaeis guineensis & Palma africana & Árbol frutal & Alimentación \\
\hline Annonaceae & Annona cherimola & Chirimoya & Árbol frutal & Alimentación \\
\hline Sapotaceae & Pouteria sapota & Zapote & Frutales & Alimentación \\
\hline Arecaceae & Bactris sp & Chonta & Árbol frutal & Industrial \\
\hline Rosaceae & Rubus glaucus & Mora & Frutales & Alimentación \\
\hline Vitaceae & Vitis vinifera & Uva silvestre & Frutales & Alimentación \\
\hline Musaceae & Musa paradisiaca & Banano & Frutales & Alimentación \\
\hline Heliconiaceae & Heliconia bihai & Bijao & Herbácea & Alimentación \\
\hline Convolvulaceae & Ipomoea batatas & Camote & Tubérculo & Alimentación \\
\hline Myrtaceae & Psidium guajava & Guayaba & Frutales & Alimentación \\
\hline Fabaceae & Inga feuillee & Guaba & Frutales & Alimentación \\
\hline Rutaceae & Citrus paradisi & Toronja & Frutales & Alimentación \\
\hline
\end{tabular}




\begin{tabular}{|l|l|l|l|l|}
\hline Bixaceae & Bixa Orellana & Achiote & Árbol frutal & Alimentación \\
\hline Sapotaceae & Pouteria caimito & Caimito & Frutales & Alimentación \\
\hline Urticaceae & Pourouma cecropiifolia & Uvillas & Frutales & Alimentación \\
\hline Malvaceae & Theobroma bicolor & Killa & Frutales & Alimentación \\
\hline Euphorbiaceae & $\begin{array}{l}\text { Caryodendron } \\
\text { orienocense }\end{array}$ & Maní de árbol & Frutales & Alimentación \\
\hline Arecaceae & Mauritia flexuosa & Morete & Frutales & Alimentación \\
\hline Moraceae & Artocarpas artrilis & Paparagua & Frutales & Alimentación \\
\hline Solanaceae & Capsicum sp & Ajín & Herbácea & Alimentación \\
\hline Rubiaceae & Alibertia patinoi & Borojo & Frutales & Alimentación \\
\hline Lauraceae & Cinnamomum verum & Canela & Árborea & Alimentación \\
\hline Lauraceae & Persea americana & Aguacate & Frutales & Alimentación \\
\hline Aquifoliaceae & Ilex guayusa & Guayusa & Árborea & Alimentación \\
\hline Sapindaceae & Nephelium lappaceum & Achotillo & Frutales & Alimentación \\
\hline Arecaceae & Cocos nucifera & Coco & Palmera frutal & Alimentación \\
\hline
\end{tabular}

\section{Riqueza específica por finca}

En la comunidad de San José, el número de especies manejadas por finca dentro del período estudiado va de 5 a 26 especies de cultivos agrícolas. Destacan principalmente tres fincas que manejan un promedio de más de 12 especies agrícolas anualmente, siendo estas las fincas 2, 6, 9 con un manejo de 18, 20 y 21 especies respectivamente. Mientras que en la comunidad de Yandia Yacu, el 28\% de las fincas, es decir dos de siete, manejan entre 1 y 10 especies agrícolas por año.

El diagnóstico sobre las especies vegetales que forman parte de la diversidad biológica de los sistemas de producción agrícola de las comunidades Shuar indica que la riqueza específica es baja, pues aporta aproximadamente el 5\% de las especies agrícolas disponibles en el país (Torres, 2010). Resultados similares se encontraron en estudios realizados en comunidades Kichwas de la amazonía ecuatoriana, donde se evidenciaron hasta 107 especies cultivadas en sistemas de chacras (Arias-Gutiérrez, 2016). Es importante señalar que un agroecosistema debe tener al menos 150 especies agrícolas para ser considerado con un alto índice de diversidad (Lores, 2008).

\section{Índice de Diversidad entre fincas}

Según el índice de Shannon-Wienner (H'), el menor valor de la diversidad se evidencia principalmente en las fincas 11 y 12 , con un valor de 0,0 , debido a que se practica monocultivo. Mientras que para las fincas 6 y 9 se tuvieron os valores más altos con 1,4 y 8,6 respectivamente, esto se debe a la distribución de las especies agrícolas que se mantienen en estas fincas. Las demás fincas se mantienen con valores de diversidad que varían de 1 a 7 (Tabla $3)$. 
Tabla 3. Índices de Margalef y Shannon-Wienner en los agroecosistemas de las comunidades Shuar

\begin{tabular}{|l|l|l|l|l|l|l|l|l|l|l|l|l|l|l|}
\hline Índices/Fincas & 1 & 2 & 3 & 4 & 5 & 6 & 7 & 8 & 9 & 10 & 11 & 12 & 13 & 14 \\
\hline Margalef & 2.4 & 6.3 & 3.9 & 1.9 & 4.6 & 9.8 & 3.4 & 2.1 & 8.0 & 4.2 & 0.0 & 0.0 & 1.1 & 3.8 \\
\hline $\begin{array}{l}\text { Shannon- } \\
\text { Wienner }\end{array}$ & 3.4 & 7.9 & 2,1 & 1.9 & 6.8 & 11.4 & 4.1 & 3.1 & 8.6 & 6.7 & 0.0 & 0.0 & 2.3 & 5.3 \\
\hline
\end{tabular}

\section{Importancia de las especies}

La importancia de las especies está dada por la frecuencia relativa acumulada de aparición en los sistemas estudiados, misma que se analizó en tres categorías: las especies perennes, los cultivos anuales y forestales.

En la comunidad de San José de Armenia se encontraron especies vegetales de mayor importancia tales como: Maíz (Zea maíz L.), yuca (Manihot esculenta Crantz), zapote (Matisia cordata Bonpl.), mango (Mangifera indica L.), guanábana (Annona muricata L.), café (Coffea arábica L.), aguacate (Persea americana Mill.), lima dulce (Citrus domesticus (L.) Burm. f.) (Tabla 4).

Tabla 4. Frecuencia relativa acumulada de cultivos anuales y perennes de la comunidad San José de Armenia

\begin{tabular}{|c|c|c|c|}
\hline \multicolumn{4}{|c|}{ Cultivos San José de Armenia } \\
\hline \multirow{2}{*}{ Perennes } & Frecuencia & \multirow{2}{*}{ Anuales } & Frecuencia \\
\cline { 2 - 2 } & $\%$ & Zea maíz L. & $\%$ \\
\hline Matisia cordata Bonpl. & 55 & Manihot esculenta Crantz. & 68 \\
\hline Mangifera indica L. & 40 & & \\
\hline Annona muricata L. & 38 & & \\
\hline Coffea arábica L. & 37 & & \\
\hline Persea americana Mill. & 35 & & \\
\hline Citrus domesticus (L.)Burm. F. & 25 & & \\
\hline
\end{tabular}

Mientras en la comunidad de Yandia Yacu encontramos diversidad de especies vegetales y forestales como: maíz (Zea maíz L.), yuca (Manihot esculenta Crantz), café (Coffea arábica L.), lima dulce (Citrus domesticus (L.) Burm. f.), y especies maderables: cedro (Cedrela odorata L.), Capirona (Calycophyllum spruceanum Benth.) (Tabla 5).

Tabla 5. Frecuencia relativa acumulada de cultivos anuales, perennes y forestales de la comunidad Yandia Yacu

\begin{tabular}{|c|c|c|c|c|c|}
\hline \multicolumn{9}{|c|}{ Cultivos Yandia Yacu } \\
\hline \multirow{2}{*}{ Perennes } & Frecuencia & \multirow{2}{*}{ Anuales } & Frecuencia & \multirow{2}{*}{ Forestales } & Frecuencia \\
\cline { 2 - 4 } & $\%$ & Zea maíz L & 70 & C.odorata L. & 38 \\
\hline Coffea arábica L. & 35 & $\begin{array}{c}\text { Manihot } \\
\text { esculenta Crantz. }\end{array}$ & 65 & $\begin{array}{c}\text { C. Spruceanum } \\
\text { Benth. }\end{array}$ & 34 \\
\hline $\begin{array}{c}\text { Citrus domesticus } \\
\text { (L.) Burm. f. }\end{array}$ & 30 & &
\end{tabular}


De esta manera se puede notar que en los cultivos anuales la especie vegetal alimenticia que predomina en la zona de estudio con una mayor frecuencia acumulada es el maíz (Zea maíz L.), con un promedio de 72,5\%, mientras que dentro de las especies perennes de mayor importancia se considera al café (Coffea arábica L.), con un promedio de frecuencia acumulada del 36\%, y la especie ancestral anual de mayor relevancia es la yuca (Manihot esculenta Crantz.) no solo por ser altamente comercial, o por hábitos alimenticios de los pobladores, sino por poseer una frecuencia acumulada del $66,5 \%$ en las zonas de cultivos.

Adicional es importante señalar que los cultivos manejados en la zona responden a una alimentación básica, con una mayor presencia de carbohidratos (Lores, 2008), presentando deficiencias nutricionales de energía, grasa, proteína y vitaminas. Esto sin duda responde también a la existencia de monocultivos e industrialización de los sistemas de producción (Franco et al., 2016). A su vez pone en riesgo la pérdida de conocimiento ancestral en la gestión y manejo de la producción y recursos de la amazonía ecuatoriana (Arias-Gutiérrez, 2016), por lo tanto es necesario incrementar la producción y conservar la diversidad de especies agrícolas en la región amazónica, considerando la interacción con la naturaleza, aprovechamiento, conocimiento ancestral, socioeconómico y demográfico (Arias-Gutiérrez, 2016). Además generar estrategias que garanticen la seguridad alimentaria de las familias, e implementar medidas de conservación, con sistemas de producción sostenibles como la agroforestería, priorizando el uso de especies nativas con alto valor comercial, que disminuyan los riesgos de erosión genética, y a su vez garanticen la conectividad del paisaje.

\section{Conclusión}

Los sistemas de cultivo ancestral y la agrobiodiversidad de la amazonía ecuatoriana, se encuentran amenazados por la intensificación de la agricultura, y presencia de monocultivos que están determinados y condicionados directamente por los hábitos alimenticios de las comunidades y principalmente dentro del contexto socio económico por la demanda en el mercado local.

La recuperación de la agrobiodiversidad en la amazonía ecuatoriana requiere emprender a nivel comunitario actividades de conservación, y enriquecimiento de las áreas de bosque que se encuentran degradadas, mediante la utilización de especies nativas amenazadas con alto valor nutricional y comercial.

\section{References:}

1. Arias-Gutiérrez, R. I., González Sousa, R., Herrera Sorzano, A., y Pérez Quintana, M. L. (2016). Agrobiodiversidad amazónica y 
estrategias de desarrollo local sostenible. Revista de Ciencias Sociales, (Cr), 1(151).

2. Camacho, E. C. (2009). Tecnologías ancestrales y su vigencia frente al cambio climático. CienciAgro, 1(4), 138-142.

3. Franco, T. L. (2003). Análisis estadístico de datos de caracterización morfológica de recursos fitogenéticos. Boletín técnico: Instituto Internacional de Recursos Fitogenéticos (8), 89.

4. Franco, W., Peñafiel, M., Cerón, C., y Freire, E. (2016). Biodiversidad productiva y asociada en el Valle Interandino Norte del Ecuador. Bioagro, 28(3), 181-192.

5. GADPO, 2015: "Plan de Desarrollo y Ordenamiento Territorial de la Provincia de Orellana 2015-2019",

6. Gutiérrez, R. I. A., Sorzano, A. H., y Sousa, R. G. (2015). El territorio indígena kichwa y el desarrollo local en la amazonia ecuatoriana. Revista de geografia agrária, 10(20), 393-415.

7. Hejda, M., Pyšek, P., y Jarošík, V. (2009). Impact of invasive plants on the species richness, diversity and composition of invaded communities. Journal of ecology, 97(3), 393-403.

8. INEC. (2010). Censo de población y vivienda en el Ecuador. Quito.

9. Lores, A., Leyva, A., y Tejeda, T.(2008). Evaluación espacial y temporal de la agrobiodiversidad en los sistemas campesinos de la comunidad "Zaragoza" en La Habana. Cultivos Tropicales, 29(1), 510.

10. Nicholls, C. I., Altieri, M. A., y Vázquez, L.L (2015). Agroecología: Principios para la conversión y el rediseño de sistemas agrícolas. Agroecología, 10(1), 61-72.

11. Nodari, R.O, y Tomás, D. F. (2016). Agrobiodiversidad y desarrollo sostenible: la conservación in situ puede asegurar la seguridad alimentaria. Biocenosis, 24(1-2), 21-29.

12. Rodríguez, C., y Castillo, M. (2005). Análisis de los Sistemas de Producción en el Territorio de Seis Comunidades Kichwas, Asentadas en la Zona Noroccidental del Parque Naciona Yasuní, Río Napo, Provincia de Orellana, Ecuador. Biodiversity \& Sustainable Forestry (BIOFOR) IQC.

13. Sarandón, S. J., y Flores, C. C. (2014). Agroecología: bases teóricas para el diseño y manejo de agroecosistemas sustentables. Colección libros de cátedra. Editorial de la Universidad Nacional de La Plata. Capítulo, 5, 131-158.

14. Somoza, A., Vázquez, P. G., \& Ruiz, D. (2015). Conservación y uso sostenible de la biodiversidad en un establecimiento rural representativo del partido de Tandil. La Plata : V Congreso Latinoamericano de Agroecología-SOCLA. 
15. Stupino, S., Iermanó, M. J., Gargoloff, N. A., y Bonicatto, M. M. (2014). La biodiversidad en los agroecosistemas. Agroecología: bases teóricas para el diseño y manejo de agroecosistemas sustentables. Colección libros de cátedra. Editorial de la Universidad Nacional de La Plata. Capítulo, 5, 131-158.

16. Torres, M. de L. (2010). Agrobiodiversidad y Biotecnología. Polémika, 5(1), 130-139. 